\title{
STATUS OF \\ MEASUREMENTS AND INTERPRETATION OF \\ TOTAL REAL AND VIRTUAL PHOTON-PROTON CROSS SECTIONS
}

\author{
CH. AMELUNG \\ (for the ZEUS and H1 Collaborations) \\ Physikalisches Institut der Universität Bonn, Nußallee 12, \\ 53115 Bonn, Germany \\ E-mail: amelung@physik.uni-bonn.de
}

\begin{abstract}
The status of measurements and interpretation of the proton structure function $F_{2}$ is summarized. The measurements are subjected to DGLAP fits, from which the gluon density is extracted and a comparison to measurements of $F_{2}^{c}$ is performed. The longitudinal structure function, $F_{L}$, is extracted via an extrapolation of $F_{2}$. At low $Q^{2}$, the transition from virtual to real photon exchange is studied.
\end{abstract}

\section{Introduction}

The proton structure functions carry information about the dynamics of quarks and gluons inside the proton, and they can be used to test the theory of the strong interaction. The measurement of the structure functions is an important part of the physics program at the ep collider HERA, and has been performed by the ZEUS and H1 collaborations.

\subsection{Kinematics of Deep Inelastic Scattering}

Inelastic positron-proton scattering, $e^{+} p \rightarrow e^{+} X$, can be described in terms of two kinematic variables, $x$ and $Q^{2}$, where $x$ is the Bjorken scaling variable and $Q^{2}$ the negative squared four-momentum transfer. In the absence of initialand final-state radiation, $Q^{2}=-q^{2}=-\left(k-k^{\prime}\right)^{2}$ and $x=Q^{2} /(2 P \cdot q)$, where $k$ and $P$ are the four-momenta of the incoming positron and proton, respectively, and $k^{\prime}$ is the four-momentum of the scattered positron. The fractional energy transfer to the proton in its rest frame, $y$, can be related to $x$ and $Q^{2}$ by $Q^{2}=s x y$, where $s=4 E_{e} E_{p}$ is the square of the positron-proton center-ofmass energy. Here, $E_{e}(27.5 \mathrm{GeV})$ and $E_{p}(820 \mathrm{GeV})$ are the positron and proton beam energies, respectively. For $Q^{2} \ll M_{Z}^{2}$, positron-proton scattering is characterized by the exchange of a photon. The photon-proton center-ofmass energy squared is defined as $W^{2}=Q^{2}(1-x) / x+m_{p}^{2}$.

The kinematic variables are closely related to the energy, $E_{e}^{\prime}$, and angle, $\theta$, of the scattered positron, where $\theta$ is measured with respect to the positron 


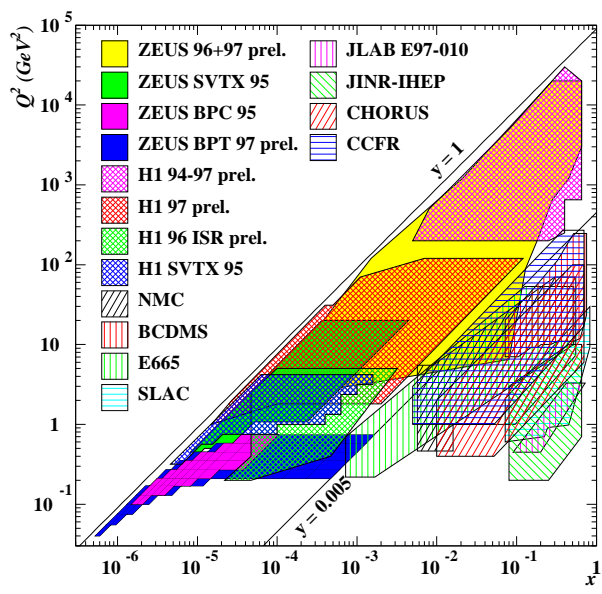

Figure 1: The range in the kinematic plane $Q^{2}$ vs. $x$ covered by the HERA experiments and by various fixed-target experiments. In most parts of the $Q^{2}$ range, ZEUS and H1 data overlap with the fixedtarget data. At low $Q^{2}$, the transition from $\gamma^{*} p$ to $\gamma p$ scattering can be studied. Measurements at medium $Q^{2}$ are used for studies and tests of pQCD. At high $Q^{2}$, electroweak effects become important and searches for new physics are performed.

beam direction: $y=1-E_{e}^{\prime} / 2 E_{e}(1+\cos \theta)$ and $Q^{2}=2 E_{e} E_{e}^{\prime}(1-\cos \theta)$.

HERA has extended the previously accessible range in the kinematic variables $x$ and $Q^{2}$ by two orders of magnitude, as shown in Fig. 1 .

\subsection{Cross Sections and Structure Functions}

The double-differential cross section for inelastic $e^{ \pm} p \rightarrow e^{ \pm} X$ scattering can be expressed in terms of structure functions as

$$
\frac{d^{2} \sigma\left(e^{ \pm} p \rightarrow e^{ \pm} X\right)}{d y d Q^{2}}=\frac{2 \pi \alpha^{2}}{y Q^{4}}\left(Y_{+} F_{2}-y^{2} F_{L} \mp Y_{-} x F_{3}\right)\left(1+\delta_{r}\right),
$$

where $Y_{ \pm}=1 \pm(1-y)^{2}$, and the radiative correction to the Born cross section, $\delta_{r}$, is a function of $y$ and $Q^{2}$, but to a good approximation independent of $F_{2}$ and $F_{L}$. From the measured quantity, the double-differential ep cross section, $F_{2}$ is extracted by making suitable assumptions about $F_{L}$ and $x F_{3}$.

At $Q^{2} \ll M_{Z}^{2}$, where $x F_{3}$ can be neglected, the $e p$ cross section can also be expressed in terms of total cross sections for the absorption of transversely and longitudinally polarized virtual photons,

$$
\frac{d^{2} \sigma\left(e^{ \pm} p \rightarrow e^{ \pm} X\right)}{d y d Q^{2}}=\Gamma\left(\sigma_{T}^{\gamma^{*} p}+\epsilon \sigma_{L}^{\gamma^{*} p}\right)\left(1+\delta_{r}\right),
$$

where $\Gamma=\alpha Y_{+} /\left(2 \pi y Q^{2}\right)$, and $\epsilon=2(1-y) / Y_{+}$. At low $x$, the photon-proton cross sections are related to the structure functions by

$$
\sigma_{\text {tot }}^{\gamma^{*} p}=\sigma_{T}^{\gamma^{*} p}+\sigma_{L}^{\gamma^{*} p} \approx 4 \pi^{2} \alpha / Q^{2} \cdot F_{2} \quad \text { and } \quad \sigma_{L}^{\gamma^{*} p} \approx 4 \pi^{2} \alpha / Q^{2} \cdot F_{L} .
$$


The so-called reduced cross section

$$
\sigma_{r}=F_{2}-\frac{y^{2}}{Y_{+}} F_{L} \mp \frac{Y_{-}}{Y_{+}} x F_{3}
$$

can also be defined, which differs from the double-differential cross section only by a kinematic factor, and can thus be extracted without assumptions about $F_{L}$ and $x F_{3}$. In most of the kinematic region covered by the present analyses, $y^{2} F_{L}$ and $Y_{-} x F_{3}$ are negligible, and thus $\sigma_{r} \approx F_{2}$.

In lowest-order QCD, $F_{2}$ can be expressed as the charge-weighted sum of quark and antiquark densities in the proton. While fixed-target experiments probe predominantly the valence-quark content of the proton, the HERA experiments are mainly sensitive to the sea contribution arising from gluon radiation off quarks and subsequent gluon splitting into quark-antiquark pairs. Only at very high $Q^{2}$ do the HERA experiments also probe the valence quarks.

\section{Structure Functions at High and Medium $Q^{2}$}

The measurement of $F_{2}$ is an inclusive cross-section measurement using the angle and energy of the detected scattered positron. Good detection efficiency and purity require both tracking and calorimetric energy measurements. The ZEUS $F_{2}$ measurement at medium and high $Q^{2}$ used the uranium-scintillator calorimeter and the central tracking detector. The acceptance of the latter, however, is limited to $16^{\circ}<\theta<165^{\circ}$. The $\mathrm{H} 1$ collaboration upgraded their detector in 1997 to improve the positron detection at small scattering angles by adding four rear discs to their microvertex detector (Backward Silicon Tracker, BST), which extend the tracking acceptance down to $3^{\circ}$.

\subsection{Measurement of $F_{2}$}

Figure 2 shows an overview of $F_{2}$ measurements at low and medium $Q^{2}$. In selected kinematic regions, ZEUS and $\mathrm{H} 1$ have achieved $F_{2}$ results with a precision of $1 \%$ (stat.) and $3 \%$ (sys.), comparable to the typical precision of fixed-target experiments.

The measured $F_{2}$ can be well described by a form $F_{2} \propto x^{-\lambda}$. The quantity $\lambda$ decreases as $Q^{2}$ decreases, corresponding to the rise of $F_{2}$ becoming less steep. As can be seen from Eq. (3), the rise of $F_{2}$ with $x$ corresponds to an energy dependence of $\sigma_{\text {tot }}^{\gamma^{*} p}$ of $\sigma_{\text {tot }}^{\gamma^{*} p} \propto W^{2 \lambda}$.

Two alternative and complementary theoretical frameworks are used to interpret these data. At medium to high $Q^{2}$, i.e. in hard interactions, pQCD is applicable, because $\alpha_{s}$ is small. At low $Q^{2}$, i.e. in soft interactions, $\alpha_{s}$ is 
HERA 1995-1997 preliminary

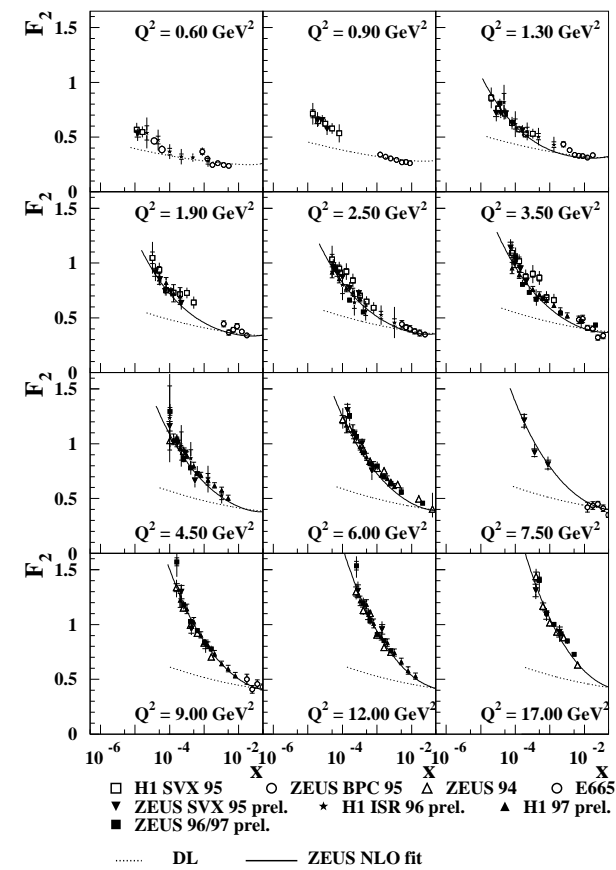

Figure 2: Overview of $F_{2}$ measurements at low and medium $Q^{2}$. The symbols denote measurements from ZEUS, H1 and E665 in different parts of the kinematic range and by special analysis techniques (SVX: shifted vertex, ISR: events with initial state radiation, BPC: using a special calorimeter close to the beam line). The solid line is the result of a DGLAP fit, the dotted line a Regge-type parqmeterization by Donnachie and Landshoff 4 .

large, and perturbative calculation techniques break down. This is the domain of Regge theory.

\subsection{DGLAP Fits}

The DGLAP equations 1 predict the evolution of parton densities with $Q^{2}$ (but make no statement about the $x$ dependence). They relate the derivative of gluon and quark densities with $Q^{2}$ to the convolution of gluon and quark densities with the splitting functions $P_{i j}$. The latter are calculable in pQCD and are a measure of the probability to find a parton $i$ radiated by a parton $j$ as a function of $Q^{2}$.

In order to compare the $Q^{2}$ evolution of parton densities as predicted by the DGLAP equations to that of the measured $F_{2}$ data, the gluon, sea quark and valence quark densities are parameterized by a suitable form at a starting scale $Q_{0}^{2}$. The parton densities are evolved to other values of $Q^{2}$, and $F_{2}$ is calculated from the quark densities. The free parameters of the parton density parameterizations are adjusted such that the resulting $F_{2}$ parameterization fits 

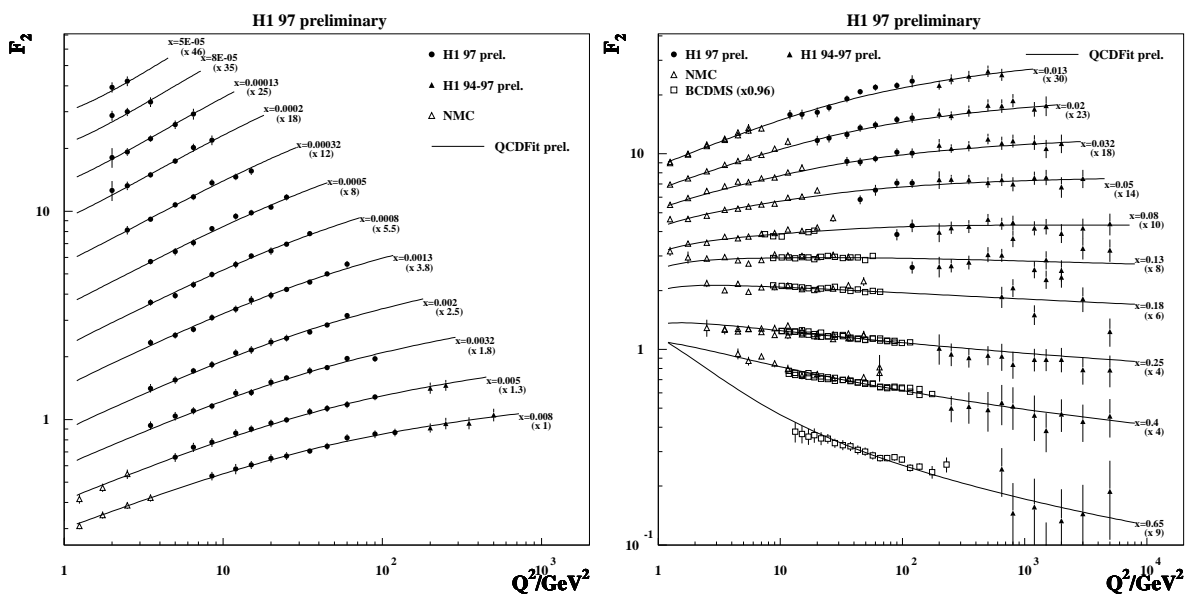

Figure 3: The scaling violations of $F_{2}$ as measured by $\mathrm{H} 1$ and the fixed-target experiments NMC and BCDMS, displayed as $F_{2}$ vs. $Q^{2}$. The solid line is the result of a DGLAP fit, which is able to describe $F_{2}$ over four orders of magnitude in $Q^{2}$.

the data above a cut-off value of $Q_{\min }^{2}$.

Figure 3 shows the result of such a fit to $F_{2}$ data from H1. The value of $F_{2}$ increases at small $x$ when increasing $Q^{2}$, while it decreases simultaneously at large $x$. The latter is a consequence of valence quarks radiating gluons and thus losing momentum, while the former results from the radiated gluons splitting into low-momentum quark-antiquark pairs. Scaling, i.e. $Q^{2}$-independence of $F_{2}$, can be seen only at $x \approx 0.1$. The fit describes the measured $F_{2}$ values well over four orders of magnitude in $Q^{2}$, which is an impressive confirmation of QCD. The fit does not deviate from the data down to the (surprisingly low) value of $Q^{2}=1 \mathrm{GeV}^{2}$, where the application of $\mathrm{pQCD}$ is somewhat questionable.

\subsection{The Gluon Density}

The gluon density can be extracted from a DGLAP fit to measured $F_{2}$ data. The result from ZEUS is shown in Fig. A. Much effort has been put into a reliable determination of the precision of this extraction, which is $10 \%-15 \%$. A comparison of the value of $x g\left(x, Q^{2}\right)$ to the value of $F_{2}\left(x, Q^{2}\right)$ at low $x$ shows that the overwhelming fraction of partons in the proton at low $x$ are gluons.

Like the quark densities, the gluon density increases with $Q^{2}$. However, when going down to $Q^{2}$ values as low as $1 \mathrm{GeV}^{2}$, the gluon density from the fit becomes compatible with zero at $x<10^{-2}$, while the sea quark density 


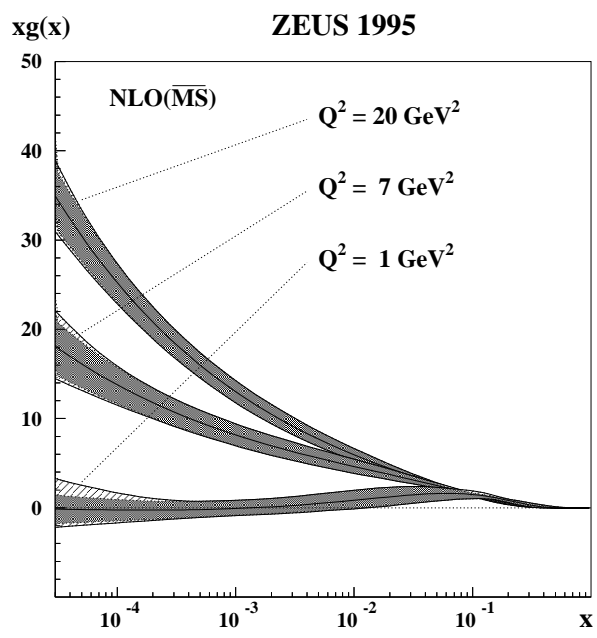

Figure 4: The gluon density resulting from a DGLAP fit to $F_{2}$ data from ZEUS, for three different values of $Q^{2}$. The shaded bands show the uncertainty of $x g\left(x, Q^{2}\right)$. At $Q^{2}=1 \mathrm{GeV}^{2}$, the gluon density as extracted from the fit becomes compatible with zero at $x<10^{-2}$.

remains positive and rises up to the smallest accessible values of $x$. This seems to be in contradiction to the concept that quarks at low $x$ are produced by gluon splitting, and may indicate a problem in applying pQCD at such low values of $Q^{2}$.

\subsection{Measurement of $F_{2}^{c}$}

The charm structure functions $F_{i}^{c}$ are defined in analogy to (1) with the requirement that the final state contains a $c \bar{c}$ pair:

$$
\frac{d^{2} \sigma\left(e^{ \pm} p \rightarrow e^{ \pm} c \bar{c} X\right)}{d y d Q^{2}}=\frac{2 \pi \alpha^{2}}{y Q^{4}}\left(Y_{+} F_{2}^{c}-y^{2} F_{L}^{c} \mp Y_{-} x F_{3}^{c}\right)\left(1+\delta_{r}\right) .
$$

At HERA, charm is generated predominantly in the photon-gluon fusion process $\gamma g \rightarrow c \bar{c}$, and is detected by reconstructing the fragmentation products of the $c$ quark, usually in the channel $D^{*} \rightarrow D^{0} \pi_{\text {slow }}$, where the $D^{0}$ decays further to $K \pi, K \pi \pi \pi$, or semi-leptonically. At the current level of precision, $F_{L}^{c}$ and $F_{3}^{c}$ can safely be neglected. In contrast to the fully inclusive $F_{2}$ measurements, further input is needed, e.g. the fragmentation function for $c \rightarrow D^{*}$ and the $D^{*} \rightarrow D^{0} \pi$ branching ratio. In addition, the event selection efficiency is quite low, because experimentally only a limited kinematic region of the $D^{*}$ is accessible. Therefore, a Monte Carlo simulation (based on the measured inclusive $F_{2}$ ) has to be used to extrapolate to the full kinematic region.

The result of the extraction of $F_{2}^{c}$ by ZEUS is shown in Fig. 5 . The charm structure function $F_{2}^{c}$ exhibits a steeper rise toward low $x$ than the fully 


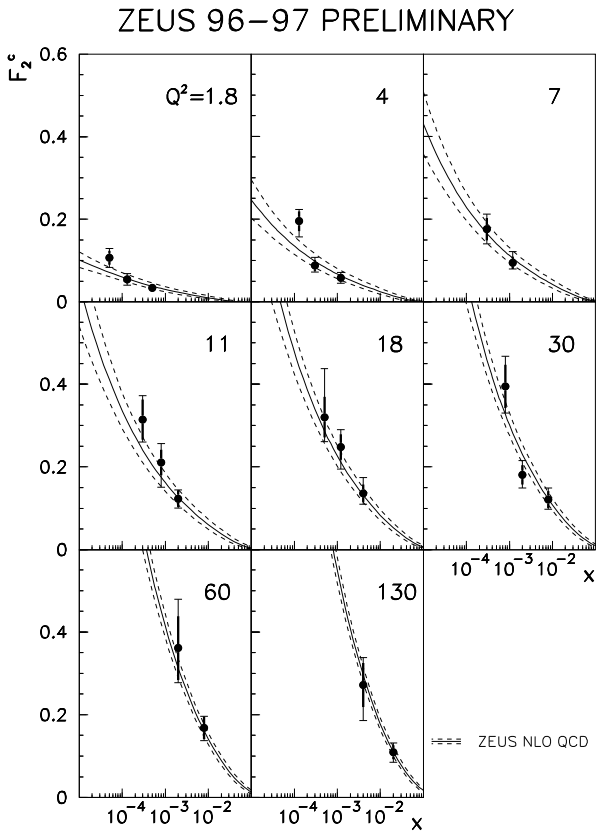

Figure 5: The charm structure function $F_{2}^{c}$ as a function of $x$ in bins of $Q^{2}$, measured by ZEUS. The solid line shows the central value of $F_{2}^{c}$ as calculated from a DGLAP fit to $F_{2}$ data, which did not include the $F_{2}^{c}$ measurements shown here. The dashed lines indicate the uncertainty of the fit result. In principle, the observed agreement of the measured $F_{2}^{c}$ with that extracted from a DGLAP fit to the inclusive $F_{2}$ is an important consistency check of the DGLAP formalism; however, the significance of this observation is reduced here due to the extrapolation technique making use of the measured inclusive $F_{2}$.

inclusive $F_{2}$, and stronger scaling violations. The charm content of the proton, $F_{2}^{c} / F_{2}$, rises from about $10 \%$ at $Q^{2}=1.8 \mathrm{GeV}^{2}$ to $25 \%$ at $130 \mathrm{GeV}^{2}$.

\subsection{Extraction of $F_{L}$}

The most direct method to measure $F_{L}$ requires a variation of the beam energies, which has not been done yet. However, $F_{L}$ can be extracted from a comparison of the measured reduced cross section $\sigma_{r}$ and an assumption about the behavior of $F_{2}$, which is derived, for example, from a DGLAP fit to $\sigma_{r}$ in the region of small $y$ (where $\sigma_{r} \approx F_{2}$, because the influence of $F_{L}$ is negligible), and subsequent extrapolation to higher values of $y$. Then, $F_{L}$ is obtained from $F_{L}=Y_{+} / y^{2}\left(F_{2}^{\text {fit }}-\sigma_{r}^{\text {measured }}\right)$.

Figure 6 shows the result of such an extraction of $F_{L}$ by the H1 collaboration. A measurement of $F_{L}$ allows to make another consistency check of the DGLAP formalism, by comparing the measured $F_{L}$ to that predicted from the parton densities in the fit. The method is experimentally challenging, because the region of high sensitivity to $F_{L}$ coincides with a region where backgrounds are high and the quality of the positron detection degrades. In addition, it relies on assumptions made in the extrapolation. 

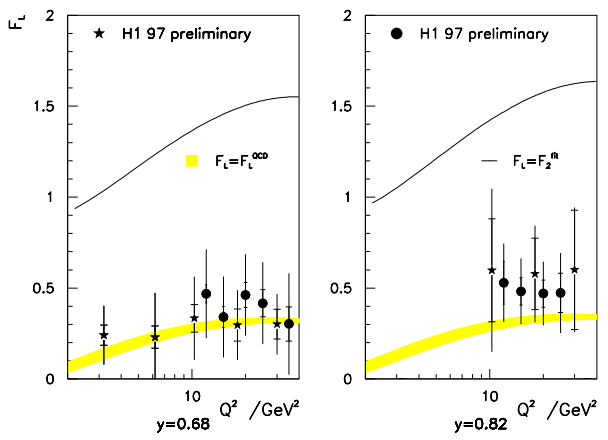

\begin{abstract}
Figure 6: Extracted $F_{L}$ from a comparison of the measured reduced cross section to an extrapolation of $F_{2}$, displayed as $F_{L}$ vs. $Q^{2}$ in two bins of $y$. Circles and stars denote results from two different extrapolation methods. Systematic errors for points in the same $y$ bin are correlated. The shaded band indicates $F_{L}$ as calculated from the result of a DGLAP fit to $F_{2}$, the solid line the upper limit $F_{L}=F_{2}$ (the lower limit is $F_{L}=0$ ).
\end{abstract}

\title{
3 Total Photon-Proton Cross Sections at Low $Q^{2}$
}

In order to study experimentally the region of very low $Q^{2}$, i.e. significantly below $1 \mathrm{GeV}^{2}$, special detectors for the scattered positron close to the beam line are necessary. The ZEUS detector was upgraded by the installation of the Beam Pipe Calorimeter (BPC) in 1995 and the Beam Pipe Tracker (BPT) in 1997, a small electromagnetic sampling calorimeter and a tracking detector of two silicon microstrip planes, detecting scattered positrons at scattering angles as low as $1.1^{\circ}-1.9^{\circ}$, thus making the region $0.045 \mathrm{GeV}^{2}<Q^{2}<0.65 \mathrm{GeV}^{2}$ accessible. H1 installed the VLQ (Very Low $Q^{2}$ ) detector, a device very similar to BPC and BPT, in 1998, and is working on the analysis of the data.

In Fig. 7 the ZEUS results of the $F_{2}$ measurement with the BPC and BPT, converted to total virtual photon-proton cross sections, are shown. The data are compared to two parameterizations by Donnachie and Landshoff (DL 1 , DL98 B), to ALLM974 and to a ZEUS Regge fit 5 of the measured data which resembles DL. The rise of $\sigma_{\text {tot }}^{\gamma^{*} p}$ with $W^{2}$ is compatible with a soft behavior for $Q^{2}$ up to $0.5 \mathrm{GeV}^{2}$, and becomes steeper at higher $Q^{2}$. At the low $Q^{2}$ values of this analysis, $\sigma_{\text {tot }}^{\gamma^{*} p}$ is nearly independent of $Q^{2}$ (in contrast, it falls like $1 / Q^{2}$ at higher $Q^{2}$ ). This can be exploited to constrain the extrapolation $\sigma_{\text {tot }}^{\gamma^{*} p} \rightarrow \sigma_{\text {tot }}^{\gamma p}$.

In order to extrapolate the measured values of $\sigma_{\text {tot }}^{\gamma^{*} p}$ to $Q^{2}=0$, an assumption about their $Q^{2}$ dependence is taken from the continuum part of the GVDMG prediction on $\sigma_{T}: \sigma_{\text {tot }}^{\gamma^{*} p}\left(W^{2}, Q^{2}\right)=m_{0}^{2} /\left(m_{0}^{2}+Q^{2}\right) \sigma_{\text {tot }}^{\gamma p}\left(W^{2}\right)$ (fitting instead both the $\sigma_{T}$ and $\sigma_{L}$ terms changes the extrapolated values only within their statistical errors).

The extrapolations are compared to the two direct measurements from ZEUS and $\mathrm{H} 1$ and to data at lower energies in Fig. 8, together with two Reggetype fits of the form $\sigma_{\text {tot }}^{\gamma p}\left(W^{2}\right)=A_{\mathbb{R}} W^{2\left(\alpha_{\mathbb{R}}-1\right)}+A_{\mathbb{P}} W^{2\left(\alpha_{\mathbb{P}}-1\right)}$, and with the DL98 and ALLM97 parameterizations. The extrapolated cross sections have 

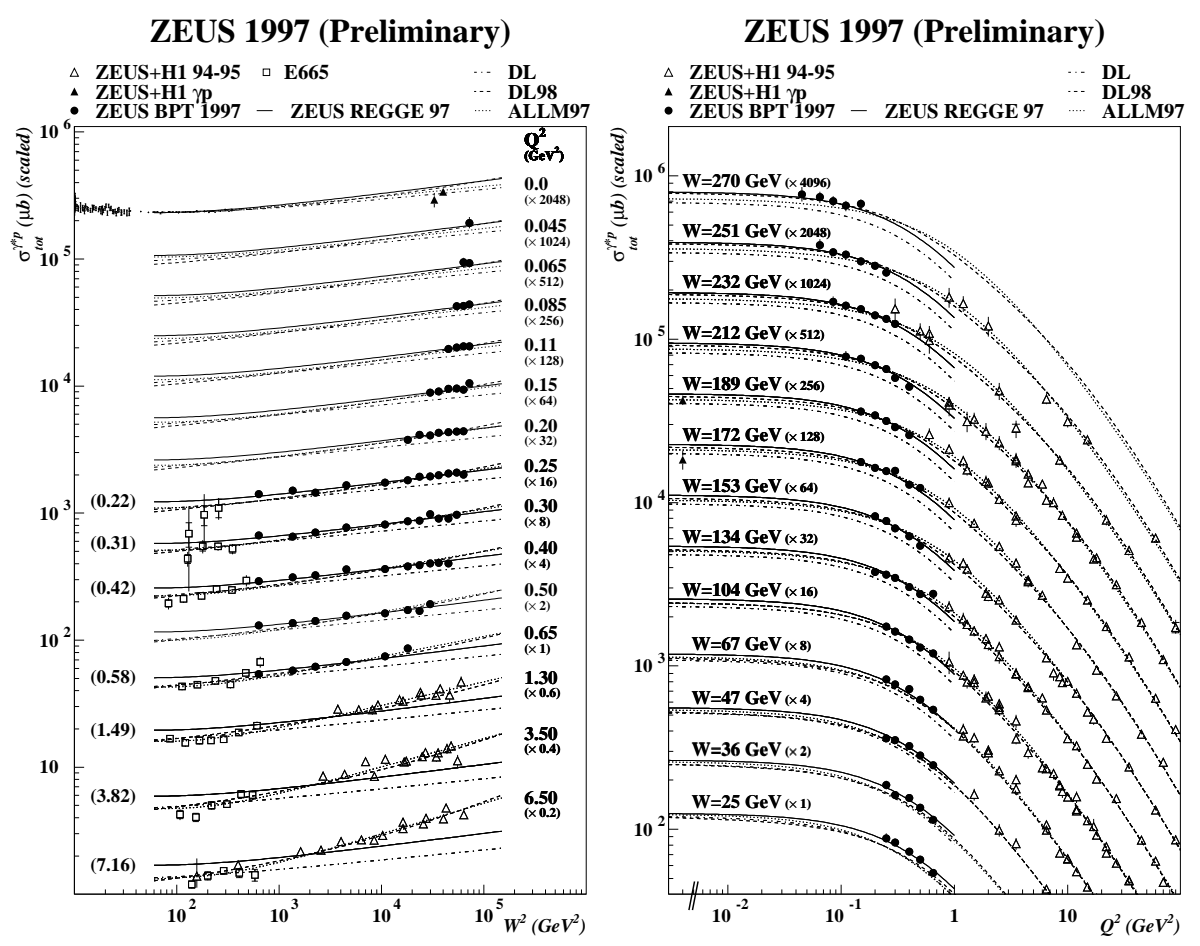

Figure 7: Measured values of $\sigma_{\text {tot }}^{\gamma^{*} p}$, plotted vs. $W^{2}$ (left) respectively $Q^{2}$ (right). Filled circles denote results from the ZEUS analysis of BPC/BPT data, filled triangles are HERA measurements at $Q^{2}=0$, open triangles represent measurements at higher $Q^{2}$, and squares denote results from E665. The data are compared to the parameterizations DL, DL98, and ALLM97, as well as to a ZEUS Regge fit of the measured data.

a tendency to be slightly higher than the directly measured ones. The modeldependence is estimated from extrapolating with the $Q^{2}$ dependence of DL, DL98 and ALLM97 (instead of GVDM), and found to be quite large at small values of $W^{2}$.

\section{Conclusions}

The HERA collaborations ZEUS and H1 have delivered a wealth of precise data on the proton structure function $F_{2}$. At $Q^{2}>1 \mathrm{GeV}^{2}$, the data can be described in a self-consistent way by DGLAP fits. At $Q^{2}$ values below $1 \mathrm{GeV}^{2}$, the transition region from the perturbative to the non-perturbative regime has been mapped. Regge-inspired models are used to describe the data here. 
ZEUS 1997 (Preliminary)

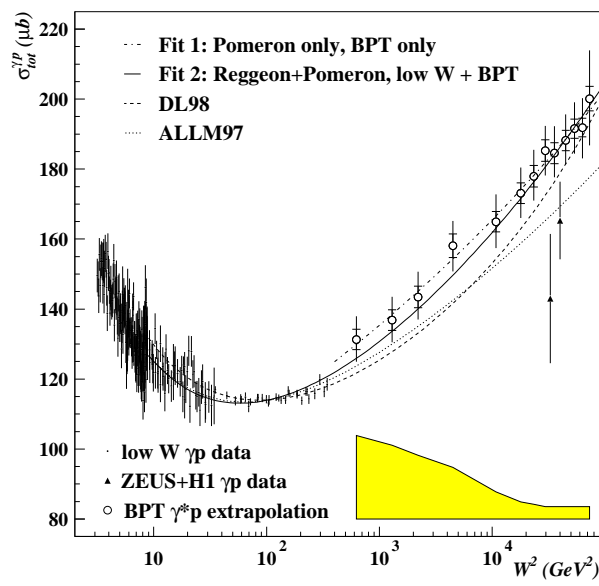

Figure 8: $\quad$ Extrapolated $\sigma_{\text {tot }}^{\gamma p}$ versus $W^{2}$ from the ZEUS analysis of BPC/BPT data, compared to the two direct measurements from ZEUS and $\mathrm{H} 1$ and to data at lower energies. The lines denote two Regge-type fits to the data, and the DL98 and ALLM97 parameterizations. The shaded band is an estimate of the magnitude of the model-dependence due to the assumed $Q^{2}$ dependence.

\section{Acknowledgments}

I would like to thank the organizers for a conference full of interesting experiences. It is a pleasure to acknowledge the outstanding effort of many colleagues in the $\mathrm{H} 1$ and ZEUS collaborations who contributed to the results presented here. I thank E. Hilger and B. Foster for careful reading of the manuscript. This work was supported by a grant from the Bundesministerium für Wissenschaft und Forschung in Germany.

\section{References}

1. A. Donnachie and P.V. Landshoff, Z. Phys. C 61 (1994) 139.

2. V.N. Gribov and L.N. Lipatov, Sov. J. Nucl. Phys. 15 (1972) 438;

L.N. Lipatov, Sov. J. Nucl. Phys. 20 (1975) 96;

G. Altarelli and G. Parisi, Nucl. Phys. B 126 (1977) 298;

Y.L. Dokshitzer, Sov. Phys. JETP 46 (1977) 641.

3. A. Donnachie and P.V. Landshoff, Phys. Lett. B 437 (1998) 408.

4. H. Abramowicz and A. Levy, DESY Report 97-251.

5. ZEUS Collaboration, J. Breitweg et al., Eur. Phys. J. C 7 (1999) 609.

6. J.J. Sakurai and D. Schildknecht, Phys. Lett. B 40 (1972) 121. 